\title{
Designing Ad-Hoc Cross Device Collaborations For Small Groups
}

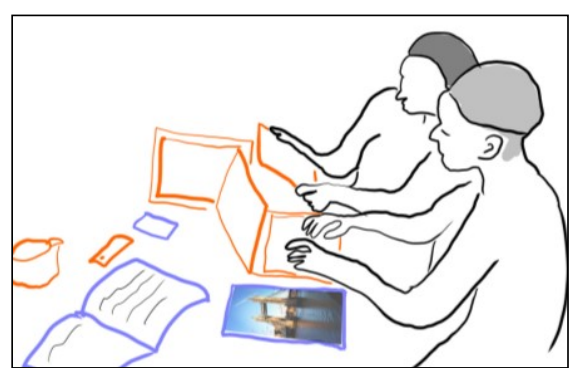

Figure 1: Ad hoc federations of people's personal mobile devices to provide cross-device interactions supporting small group-collaboration when curating digital and non-digital historic documents.

\section{Frederik Brudy}

UCL Interaction Centre, University College London

66 - 72 Gower Street, London WC1E 6EA, UK

f.brudy@cs.ucl.ac.uk

\section{Abstract}

The curation of historic documents is a difficult task as it requires to combine information and raw material from many different sources. Digital tools can support such a sensemaking task and group collaboration can help the discovery of knowledge. While most of people's personal devices (such as phones, tablets and laptops) are connected to the internet, they are not aware of each other's presence or relationship when in close proximity. Leveraging people's personal devices and other devices in their surroundings provides an opportunity to support the curation of historic documents in ad hoc small group scenarios.

I describe my motivation and a selection of related work, leading to requirements for such a system. I then Permission to make digital or hard copies of part or all of this work for personal or classroom use is granted without fee provided that copies are not made or distributed for profit or commercial advantage and that copies bear this notice and the full citation on the first page. Copyrights for third-party components of this work must be honored. For all other uses, contact the Owner/Author.

Copyright is held by the owner/author(s).

ITS '15, November $15-18,2015$, Funchal, Portugal

ACM $978-1-4503-3899-8 / 15 / 11$.

http://dx.doi.org/10.1145/2817721.2820984 state how I am planning to address these challenges and my current state of research, following two paralle tracks: building and testing technology as well as conducting observational studies and interviews to inform my designs.

\section{Author Keywords}

Cross device interaction; ad-hoc collaboration; small group collaboration; curation of historic documents and artefacts

\section{ACM Classification Keywords}

H.5.3. Group and Organization Interfaces. Computersupported cooperative work; H.5.2. User Interfaces. Input devices and strategies

\section{Introduction}

Analyzing and curating historic documents in order to prepare them for further use (for example in print or online) requires to gather, review and combine large collections of documents and artefacts, making this a demanding task [9]. With growing number of items in such collections and various different document types and formats, digital technology is needed in order to support this. Furthermore, collaboratively curating these collections in a small group can make it easier to reveal interesting facts and find new connections between them (Figure 1). Previous research explored 
how a single person can do this, e.g. on large screens [1], tabletops [3] or using multiple devices [4]. However most of our devices do not well support such a collaborative task: although we are surrounded with smart devices, such as smartwatches, smartphones, tablets, laptops, and large wall and tabletop displays, we are primarily using them for individual tasks to collect, study and analyze information. Even though these devices can communicate with other devices, they are not aware of their immediate presence, nor of their spatial or semantic relationship. They do not support ad hoc collaborations, e.g., through sharing of content across devices (such as photos, historic maps, video and text) and display space.

I believe that there is a potential for small groups to use their existing devices when analyzing a pool of documents. Therefore I have begun my work with a group of volunteer historians, learning from their curation activities in order to support them with digital technology.

\section{Related work}

Sensemaking, as used in Human Computer Interaction $(\mathrm{HCI})$, is the process of analyzing collections of information in order to find common activities, make an understanding and process the data for further use [10]. When compared to analyzing documents on a computer, paper's physical affordance makes for three unique properties, supporting this task [7]:

- Annotation. Paper documents can be easily annotated, which is integrated into reading.

\section{- Movement within and between documents.}

Moving quickly through large amounts of documents, while skimming over content is easier with paper.
- Spatial layouts. Documents can be arranged in space and laid out representing a structure that supports the knowledge discovery, e.g. grouping documents by topic or arranging them in a timeline.

With an increasing amount of data, doing this with paper documents becomes more tedious (or even impossible) and not all information can be acquired in a printed format (e.g., as in case of video or audio this is simply not possible without further processing). With other formats, richness of information or the context of it will be lost (e.g., location data embedded in a photo). Computer systems can support this, e.g. through search tools, visualization and isolation of content and connection, and finding related content. However it is left to the human to fully understand the meaning and draw connections.

Using large tabletops or wall displays to curate such collections has been proposed and cross-device interactions have been in the focus of HCI research since a few years. Andrews et al. explored how large, high-resolution displays support sensemaking tasks [1]. An example for a system that allows to distribute resources and services across multiple devices is ActivitySpace [5]. Conductor [4] is a single-user multidevice system enabling a person to use up to 10 personal devices in a sensemaking task. A group's appropriation of the space on and around a tabletop display has been described [14], Ryall et al. reported about how a group's strategies and speed are affected by group and table size [11] and MultiSpace [3] explores how a tabletop and wall-display supports ad hoc collaboration in a multi-device environment. However these devices and systems are expensive to buy, need a long setup process, require a permanent place where they are set up or do not support 
sensemaking with multiple people. They are not suitable for ad hoc curation of documents in small groups.

On the contrary we carry more and more mobile but computationally powerful devices with us. Previous research explored the spatial relationships of devices (e.g. $[2,8,15])$, cross device interactions which support changing numbers of devices (e.g. $[4,8]$ ), the use of personal devices to bring in information (e.g. [12]), as well as body worn devices in concert with wall displays (e.g. [16]). More recently with HuddleLamp [8] a multiuser multi-device framework has been proposed,

allowing for spatial tracking of tablets and phones on a table and for cross device interactions.

In summary, a system for sensemaking and curation of documents should support the following features:

- Portability. A system should be easy to setup, without the requirement for a permanent place. This would allow for usage in various spaces.

- Ad hoc. Supporting a walk-up-an-go collaboration, without a tedious setup and long preparation works.

- Low cost. In order to be easily deployable a system should be either available to work with existing devices or only require low initial costs.

- Multi-user. In order to support small-group sensemaking and curation of documents, a system should support collaborative multi-user scenarios.

- Heterogeneous data and environments. To account for the rich nature of documents in data collections, a system should account for both, digita and non-digital content and allow to work in environments with cluttered work areas and noisy data.

\section{Research objectives}

My research addresses the question how we can support the curation of historic documents in small group scenarios with digital technology, using people's personal devices and the devices in their surroundings. I divide my work into three sections.

1. Study people's current practices. This will build a framework for group collaborations for ad hoc small group scenarios. Some of the questions I am trying to answer include the following: How do people make use of devices and utilities (paper, pens, tablets, screens, etc.)? How is material and content shared when a group meets to discuss or work on a topic? How are devices being held during particular tasks, e.g. a collaborative research task about a historic event, during office works or when preparing for a class presentation at school?

2. Develop technology. In order to support the aforementioned small group collaborations I am building a tool, allowing small groups to curate documents in ad hoc scenarios, leveraging people's personal and publicly available devices. I have started with individual prototypes, which try to solve a single requirement, e.g. digitizing of physical artifacts or temporary sharing of content in small groups.

\section{Deploy and evaluate more collaborative}

technology. In a third step I will broaden up the area to which I am applying my technologies and open it up to more use cases (rather than only curation of historic documents). This will broaden up and support the framework of objective 1 .

Each of these sections should not be seen as a single unit, nor will they be in a sequential order. Rather each unit will influence and inform the others throughout my work in an iterative design process. For example the lessons learned from unit 1 will help me to understand current collaboration techniques and thus to build the right tools 


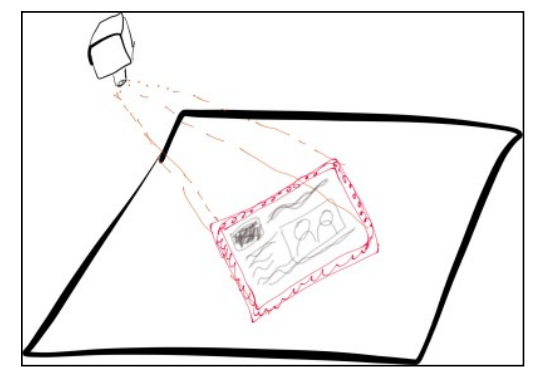

Figure 2: The Tangible Scanner allows to digitize physical documents, in order to support working with physical and digital objects.

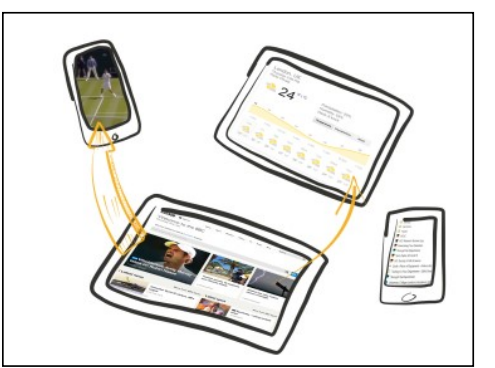

Figure 3: Content can be distributed across multiple devices (here: content can be flicked between two tablets and two phones). Device's position and orientation is tracked using HuddleLamp, allowing for a shared canvas and cross-device interactions.

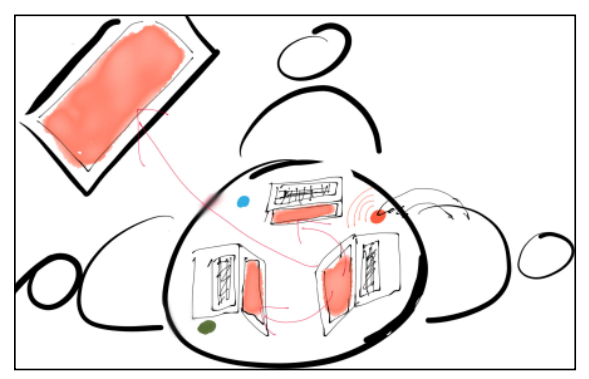

Figure 4: The meeting presenter allows to share content in a meeting with the push of a button. to support these. I will test these either by deploying them to my target audience or in lab studies.

I have started to follow two tracks in parallel. On one side I am exploring the technological aspects and the potential of cross-device interactions, which will allow me to explore the potential of using everyday devices in ad hoc small group collaborations, leveraging cross-device sharing of e.g., photos, text, videos and more. For this I am using technology probes prototypes [6] to present ideas and test them with collaborators. My vision for these tools

is that they should be easy to setup, work with existing or low-cost devices and allow to blend physical and digital in order to support

heterogeneous data. For example a Tangible Scanner (Figure 2) allows to easily digitize physical artefact on a table: a paper map is placed in a physical frame and the system takes a pictures of the map, making it immediately available on one of the devices on the table e.g., a person's tablet. The digital version can then be reviewed on that tablet, copied to another tablet or presented to others (Figure 4)

Distributing content across multiple devices will allow to leverage the spatial properties of them. E.g., laying multiple devices next to each other to build a timeline or stacking multiple devices to group items together, much as it is already done with paper [7] and when using large digital displays [1]. Using different form factors of devices, such as tablets, phones, large screens, laptops and even wearable devices different document formats can be accounted for. Each device will not be assigned a certain content type per-se, rather people using it can appropriate the system in a way that supports their work: a video might be viewed better on a tablet, while a phone displays a list of bookmark which, when clicked, opens a document

\footnotetext{
${ }^{1} \mathrm{http}: / /$ www.capturingcambridge.org/
}

on a large display (Figure 3).

On the other side I am conducting observational studies and interviews in order to inform my designs. I have

begun a collaboration with the Mill Road History Project in Cambridge ${ }^{1}$ (MRHP), where volunteers record, learn, curate and present the rich history of this particular street of the city. This work is performed by members of the community (most are volunteers) and accessible by everyone. Depending on the historic data, many different document types are used: historic maps and photos, audio recordings of oral histories, paper documents, location data, books, personal memories and many more. Most of the participants in this project own and use a smartphone on the go, many of them also a tablet and laptop. They meet in various spaces, without a permanent room where they could set up such a system.

The goal of my research is to foster sharing and exploring of digital content across multiple devices when they are in close proximity to one another to support group sensemaking tasks. These interactions do not have to be limited to phones and wall displays. Extending this interaction space to a user's body through wearable devices could allow for a whole new set of interaction and would give new opportunities to exchange information between users, devices and their surroundings.

\section{Current state of research}

I have conducted semi structured interviews with members of the MRHP, where I have learned about their current practices of gathering documents from various sources, how they keep track of their sensemaking status and how information is shared between members of the group. They have divided up 
their work on Mill Road into individual building reports, where one or more members of the group research the history of a particular building on the street. They prefer working with digital documents, as opposed to physical ones, allowing them to easily share them with other members of the group without having to worry about archiving after the project has ended or handing them over when someone drops out of the group. However this makes reviewing of documents more difficult as they cannot be grouped and annotated as easily as paper documents. Some members of the group have trouble with existing tools for editing, sharing and collaboratively working on documents and they end up as a group sitting in front of one laptop in order to edit a document, as the co-presence allows for immediate sharing of knowledge and helps when merging multiple sets of information. When a new person joins a team, information is still handed over in personal meetings, as this allows them to go through the documents together and explain any connections. However, the actual sensemaking in a group rarely happens. This might be because of a lack of tools which could support their current practices, although this will need further exploration.

I have also built technology that supports sharing of content across devices; capturing of physical artefacts, blending them with digital content (Figure 2); separating content across multiple devices (Figure 3); and sharing content in a meeting with the push of a button (Figure 4). Throughout I am deploying the built technologies to my collaborators in order to gather early feedback, to learn how small tools are appropriated and how they could feed into a bigger system.

\section{Further research}

I will extend my observations to small group meetings in everyday work life. This will help me understand how people share material deliberate and implicit, what kind of props are being used (such as paper, laptops, tablets, screens, etc.) and how devices are being held and used. I will build upon previous research [13] to deepen the understanding of how meetings are being held so far and to learn what a digital system has to provide in order to support them.

I will deploy a system to members of the MRHP. This tool allows them to explore the digital collections of objects, using various input mechanisms, e.g., gesture recognition, touch based interactions, and spatial arrangements of devices (e.g., their position and orientation to each other on the table). Using this system, objects can be moved across device boundaries (copy, move and flick content between e.g. tablets, phone and displays). Through quantitative measurements and observations on how they appropriate the tool I will inform my further research.

\section{Summary}

Growing collections of historic documents makes their curation almost impossible to be done with paper documents only, leading for this to happen on digital devices. Using multiple devices, the physical properties can be used in a similar way as it is with paper: they can be laid out to build a timeline, stacked to group or set aside to offload documents not currently needed. Although most of our digital devices are connected to the internet, they do not communicate to each other when in close proximity, nor are they aware of each other's presence. The overall goal of my research is to enable sharing and exploring of digital content across multiple devices to support collocated group sensemaking tasks. For this I have begun my work with a group of volunteers, curating the history of a street in Cambridge, learning from their current practices and understanding their requirements for 
their work. I am iteratively building and testing prototype systems. This allows me to explore the technical aspects and will inform the design of a system supporting the curation of documents across devices in ad hoc smallgroup collaborations.

\section{Acknowledgements}

I wish to thank my supervisors Nicolai Marquardt and Yvonne Rogers (University College London) as well as Abigail Sellen and Kenton O'Hara (Microsoft Research Cambridge).

This work is supported by Microsoft Research through its PhD Scholarship Programme.

\section{References}

[1] Andrews, C., Endert, A., and North, C. Space to think: large high-resolution displays for sensemaking. Proc. CHI, ACM (2010), 55-64.

[2] Ballendat, T., Marquardt, N., and Greenberg, S. Proxemic interaction: designing for a proximity and orientation-aware environment. Proc. ITS, ACM (2010), 121-130.

[3] Everitt, K., Shen, C., Ryall, K., and Forlines, C. MultiSpace: Enabling electronic document micromobility in table-centric, multi-device environments. TableTop 2006, IEEE (2006), 8-pp.

[4] Hamilton, P. and Wigdor, D.J. Conductor: enabling and understanding cross-device interaction. Proc. CHI, ACM (2014), 2773-2782.

[5] Houben, S., Tell, P., and Bardram, J.E. ActivitySpace: Managing Device Ecologies in an Activity-Centric Configuration Space. Proc. ITS (2014), 119-128.

[6] Hutchinson, H., Bederson, B.B., Druin, A., et al. Technology Probes: Inspiring Design for and with Families. Proc. CHI, ACM (2003), 17-24.
[7] O'Hara, K. and Sellen, A. A comparison of reading paper and on-line documents. Proc. CHI, ACM (1997), 335-342.

[8] Rädle, R., Jetter, H.-C., Marquardt, N., Reiterer, H., and Rogers, Y. HuddleLamp: Spatially-Aware Mobile Displays for Ad-hoc Around-the-Table Collaboration. Proc. ITS, ACM (2014), 45-54.

[9] Rotman, D., Procita, K., et al. Supporting content curation communities: The case of the Encyclopedia of Life. JASIST 63, 6 (2012), 1092-1107.

[10]Russell, D.M., Stefik, M.J., Pirolli, P., and Card, S.K. The cost structure of sensemaking. Proc. InterCHI, ACM (1993), 269-276.

[11]Ryall, K., Forlines, C., Shen, C., and Morris, M.R. Exploring the effects of group size and table size on interactions with tabletop shared-display groupware. Proc. CSCW, ACM (2004), 284-293.

[12] Schmidt, D., Seifert, J., Rukzio, E., and Gellersen, $\mathrm{H}$. A cross-device interaction style for mobiles and surfaces. Proc. DIS, ACM (2012), 318-327.

[13] Scott, S.D., Carpendale, M.S.T., and Inkpen, K.M. Territoriality in collaborative tabletop workspaces. Proc. CSCW, ACM (2004), 294-303.

[14]Tang, A., Tory, M., Po, B., Neumann, P., and Carpendale, S. Collaborative coupling over tabletop displays. Proc. CHI, ACM (2006), 1181-1190.

[15] Vogel, D. and Balakrishnan, R. Interactive public ambient displays: transitioning from implicit to explicit, public to personal, interaction with multiple users. Proc. UIST, ACM (2004), 137-146.

[16]von Zadow, U., Büschel, W., Langner, R., and Dachselt, R.I.M.L. SleeD: Using a Sleeve Display to Interact with Touch-sensitive Display Walls. Proc. ITS, ACM (2014), 129-138. 